\title{
Effect of Recepteur d'Origine Nantais expression on chemosensitivity and tumor cell behavior in colorectal cancer
}

\author{
NURI KIM ${ }^{1,2^{*}}$, SUNG-BUM CHO $^{1 *}, Y_{\text {OUNG-LAN PARK }}^{1}$, SUN-YOUNG PARK $^{1}$, EUN MYUNG $^{1}$, SEUNG-HUN KIM $^{1}$, \\ HYUNG-MIN YU ${ }^{1}$, YOUNG-AE SON ${ }^{1}$, DAE-SEONG MYUNG ${ }^{1}$, WAN-SIK LEE ${ }^{1}$ and YOUNG-EUN JOO ${ }^{1,2}$ \\ Departments of ${ }^{1}$ Internal Medicine and ${ }^{2}$ Biomedical Science, Chonnam National University Medical School, \\ Dong-ku, Gwangju 501-757, Republic of Korea
}

Received October 26, 2015; Accepted December 3, 2015

DOI: $10.3892 /$ or.2016.4721

\begin{abstract}
Recepteur d'Origine Nantais (RON) expression is known to induce oncogenic properties including tumor cell growth, survival, motility, angiogenesis and chemoresistance. In the present study, we evaluated whether RON affects chemosensitivity and oncogenic behavior of colorectal cancer cells and investigated its prognostic value in colorectal cancer. To evaluate the impact of RON on chemosensitivity and tumor cell behavior, we treated colorectal cancer cells with small interfering RNAs specific to RON. This was followed by flow cytometric analyses and migration, Matrigel invasion and endothelial tube formation assays. The expression of RON was investigated by immunohistochemistry in colorectal cancer tissues. TUNEL assay and immunohistochemical staining for CD34 and D2-40 were deployed to determine apoptosis, angiogenesis and lymphangiogenesis. RON knockdown enhanced 5-fluorouracil (FU)-induced apoptosis by upregulating the activities of caspases and expression of proapoptotic genes. Moreover, it enhanced 5-FU-induced cell cycle arrest by decreasing the expression of cyclins and cyclin-dependent kinases and inducing that of p21. Furthermore, RON knockdown augmented the 5-FU-induced inhibition of invasion and migration of colorectal cancer cells. The $\beta$-catenin signaling cascade was blocked by RON knockdown upon 5-FU treatment. RON knockdown also decreased endothelial tube formation and expression of VEGF-A and HIF-1 $\alpha$ and increased angiostatin expression. Furthermore, it inhibited lymphatic endothelial cell tube formation and the expression of VEGF-C and COX-2. RON expression was observed to be associated with age, tumor size, lymphovascular and perineural
\end{abstract}

Correspondence to: Professor Young-Eun Joo, Department of Internal Medicine, Chonnam National University Medical School, 8 Hak-Dong, Dong-ku, Gwangju 501-757, Republic of Korea E-mail: yejoo@chonnam.ac.kr

*Contributed equally

Key words: Recepteur d'Origine Nantais, angiogenesis, chemosensitivity, colorectal neoplasm invasion, tumor stage, lymph node and distant metastasis, and poor survival rate. The mean microvessel density value of RON-positive tumors was significantly higher than that of RON-negative ones. These results indicate that RON is associated with tumor progression by inhibiting chemosensitivity and enhancing angiogenesis in colorectal cancer.

\section{Introduction}

Colorectal cancer is one of the common causes of cancerrelated deaths worldwide. Although recent advances in early diagnosis and treatment have improved the clinical outcome in patients with colorectal cancer, recurrence and metastasis are still common (1-3). Therefore, a greater understanding of the molecular mechanisms underlying tumor recurrence and metastasis has proven useful in the management of colorectal cancer (4).

In patients with various types of cancers, cancer cells in primary tumors disseminate to distant organs, through the blood and lymphatic circulatory systems by metastasis $(5,6)$. Dysregulated angiogenesis and lymphangiogenesis occur in a variety of pathological settings including growth and dissemination of tumors (7-11). Several lines of evidence indicate that progression of colorectal cancer depends on angiogenesis and lymphangiogenesis (12-14).

The chemosensitivity of circulating cancer cells is suggested to determine chemotherapeutic response in cancer patients. Chemoresistance enables cancer cells to evade apoptotic stimuli and leads to poor clinical prognosis (15-19). Recent studies showed a correlation between poor treatment outcome and development of chemoresistance in advanced colorectal cancer patients (20-22).

Recepteur d'Origine Nantais (RON) is a member of the c-MET protooncogene family of receptor tyrosine kinases (RTKs) that has been implicated in regulating invasive growth phenotypes in a variety of cancers (23-29). Specifically, activation of RON mediates several biological events including cell growth, motility, angiogenesis, epithelial-to-mesenchymal transition and drug resistance in cancer cells (23-29). Overexpression of RON has been reported in various cancer tissues including that of the colon (30-37). Furthermore, its overexpression correlates with aggressive disease and poor prognosis in patients with colorectal, gastric or breast 
cancers (30-33). Therefore, activation of RON may be critical to the development and progression of cancers. However, the impact of RON on chemosensitivity, angiogenesis and lymphangiogenesis in colorectal cancer is yet to be elucidated.

The aims of the present study were to evaluate whether RON affects chemosensitivity and oncogenic behavior of colorectal cancer cells, and delineate the relationship between its expression and various clinicopathological parameters in colorectal cancer.

\section{Materials and methods}

Patients and tissue sample collection. Colorectal cancer tissues were obtained from 88 patients who consecutively underwent surgery for colorectal cancer at the Chonnam National University Hwasun Hospital between January and December 2006. Pathological studies and clinical histories recorded at the time of surgery were reviewed. Tissue blocks that clearly showed the junction between normal colon epithelium and tumoral region were selected by microscopic analyses of their original pathologic slides. The tumors were staged at the time of surgery using standard protocol for tumor-node-metastasis (TNM) staging given by the American Joint Committee on Cancer. Survival was tracked from the time of surgery until follow-up on December 31, 2014.

Cell culture and siRNA transfection. Human colorectal cancer cell line, DLD1 was obtained from the American Type Culture Collection Line Inc., and were maintained in Dulbecco's modified Eagle's medium (DMEM) supplemented with 10\% fetal bovine serum (both from HyClone, Logan, UT, USA). Human umbilical vein endothelial cells (HUVECs; Lonza, Walkersville, MD, USA) and human lymphatic endothelial cells (HLECs; ScienCell, San Diego, CA, USA) were maintained in EGM $^{\circledR}-2$ MV SingleQuots ${ }^{\circledR}$ (Lonza). The RON-specific and scrambled small interfering RNA (siRNA) duplexes were purchased from Santa Cruz Biotechnology (Santa Cruz, CA, USA) and Qiagen (Germantown, MD, USA), respectively. The siRNAs were transfected using Lipofectamine ${ }^{\text {TM }}$ RNAiMAX (Invitrogen, Carlsbad, CA, USA) according to the manufacturer's instructions. To obtain the conditioned medium (CM), transfected cells were incubated in serum-free medium for $24 \mathrm{~h}$.

5-Fluorouracil (FU) treatment. DLD1 cells were seeded onto 6 -well plates at densities of $3 \times 10^{5}$ cells/well, and transfected with either RON-specific or scramble siRNA. Following incubation for 24 h, 5-FU (Julian ${ }^{\circledR}$; Dongkook, Korea) was added at concentrations ranging from 0 to $80 \mu \mathrm{g} / \mathrm{ml}$ and incubated for $24 \mathrm{~h}$.

Cell proliferation assay. Cell proliferation was determined using the EZ-CyTox (tetrazolium salts, WST-1) cell viability assay kit (Daeil Lab Inc., Seoul, South Korea). Transfected DLD1 cells were treated with 5-FU at concentrations ranging from 0 to $80 \mu \mathrm{g} / \mathrm{ml}$, incubated for $24 \mathrm{~h}$ and treated with the WST-1 reagent. This was followed by incubation for $1 \mathrm{~h}$ at $37^{\circ} \mathrm{C}$. The absorbance was measured using a microplate reader (Infinite M200) equipped with Magellan V6 data analysis software (both from Tecan, Austria GmbH, Austria).
Apoptosis analysis. Transfected cells treated with 5-FU were collected using trypsin and resuspended in binding buffer (BD Biosciences, San Diego, CA, USA). The cell suspensions were then incubated with APC Annexin V and 7-amino-actinomycin D (7-AAD; BD Biosciences). The cells were gently vortexed and incubated in dark for $20 \mathrm{~min}$ at room temperature. The population of Annexin V-positive cells were analyzed using BD Cell Quest ${ }^{\circledR}$ version 3.3 (Becton-Dickinson, San Jose, CA, USA) and WinMDI version 2.9 (The Scripps Research Institute, San Diego, CA, USA).

Cell cycle analysis. Transfected cells treated with 5-FU were fixed in $70 \%$ ethanol and washed with phosphate-buffered saline (PBS). Next, the cells were incubated with $100 \mu \mathrm{g} / \mathrm{ml}$ each of ribonuclease A and propidium iodide (both from Sigma-Aldrich, St. Louis, MO, USA) at room temperature in the dark. The cell cycle profiles were analyzed using BD CellQuest ${ }^{\circledR}$ version 3.3 and WinMDI version 2.9.

Transwell invasion assay. Invasion assays were performed using Transwell filter chambers $(8.0-\mu \mathrm{m}$ pore size; Costar, Cambridge, MA, USA) with gelatin coating. The transfected, 5-FU-treated cells were seeded at densities of $2 \times 10^{5}$, with $0.2 \%$ bovine serum albumin (BSA) medium in the upper chambers. The lower chambers were filled with $0.2 \%$ BSA medium containing fibronectin $(10 \mu \mathrm{g} / \mathrm{ml})$. After $24 \mathrm{~h}$ of incubation, invading cells on the lower surface of the upper chamber were fixed with $70 \%$ ethanol and stained with Diff-Quik solution (Sysmex, Kobe, Japan) following the manufacturer's protocol. The stained cells were enumerated in five randomly selected fields under a light microscope.

Cell migration assay. Transfected, 5-FU-treated cells were seeded at densities of $1 \times 10^{5}$ culture inserts $\left(2 \times 0.22 \mathrm{~cm}^{2}\right.$; Ibidi, Regensburg, Germany). Following incubation for $24 \mathrm{~h}$, the inserts were gently removed using sterile tweezers. Cells migrated into the scratch were photographed at 0,3,6, 12 and $24 \mathrm{~h}$ time-points using an inverted microscope. Distance between the gaps was normalized to $1 \mathrm{~cm}$ after capture of three random sites.

Western blotting. Total proteins extracted with M-PER ${ }^{\circledR}$ mammalian protein extraction reagent (Thermo, Rockford, IL, USA) were resolved by sodium dodecyl sulfate-polyacrylamide gel electrophoresis and transferred onto PVDF membranes (Millipore, Billerica, MA, USA). Specific proteins were blotted with respective primary antibodies, which are as follows: antiRON, anti-cyclooxygenase-2 (COX-2), anti-vascular endothelial growth factor (VEGF)-A, -C and -D, anti-GAPDH, and anti- $\beta$ tubulin (Santa Cruz Biotechnology). Antibodies against cleaved and intact forms of caspase-3 and -9, and poly(ADP-ribose) polymerase (PARP), Bcl-xL, Bax, Bim, cyclin B1, D1 and D3, cyclin-dependent kinase (CDK) 2, 4 and 6, p21, p27, hypoxia-inducible factor-1 $\alpha$ (HIF-1 $\alpha$ ), and total and phospho$\beta$-catenin (Ser33/37/Thr41) were purchased from Cell Signaling Technology (Danvers, MA, USA). Antibodies against angiostatin and endostatin were purchased from Abcam (Cambridge, UK). The target proteins were detected using the enhanced chemiluminescence detection system horseradish peroxidase (HRP) substrate (Millipore) and luminescent image analyzer LAS 4000 (Fujifilm, Tokyo, Japan). 
In vitro tube formation assay. Matrigel basement membrane matrix (BD Biosciences) was used to coat culture plates according to the manufacturer's instructions. Approximately $50 \mu 1$ of thawed Matrigel was applied to each well of a 96-well plate and left to polymerize at $37^{\circ} \mathrm{C}$ for $1 \mathrm{~h}$. Transfected HUVECs and HLECs $\left(2 \times 10^{4}\right.$ cells/well) in $100 \mu \mathrm{l} \mathrm{CM}$ were seeded on the substratum of the Matrigel, and incubated for $24 \mathrm{~h}$ at $37^{\circ} \mathrm{C}$ and $5 \% \mathrm{CO}_{2}$. Next, the tube-like structures were photographed using an inverted microscope. The tube lengths were measured using the WimTube image analysis platform (Wimasis $\mathrm{GmbH}$, Munich, Germany) on the Ibidi website (www.ibidi.com).

Matrigel invasion assay. Transwell filters $(8.0-\mu \mathrm{m}$ pore size) were coated with $1 \mathrm{mg} / \mathrm{ml}$ of Matrigel, and dried at room temperature. Either HUVECs or HLECs were resuspended in $120 \mu \mathrm{EGM}^{\circledR}-2 \mathrm{MV}$ Single Quotes ${ }^{\circledR}$ medium, and inoculated into the upper chambers of the Matrigel-coated Transwells. The lower chambers were filled with CM from transfected cells. After incubation for $3 \mathrm{~h}$, cells on the Transwell were stained with Diff-Quik and non-invading cells on the upper surface of the Transwell were wiped off with a cotton swab. The numbers of invaded cells were enumerated in five randomly selected fields under a light microscope. Data from three independent experiments are expressed as the mean \pm standard deviation (SD) of the number of cells/field.

Immunohistochemistry. Paraffin-embedded tissue sections were deparaffinized, rehydrated and retrieved. To block the endogenous peroxidase activity, tissues were treated with peroxidase-blocking solution (Dako, Carpinteria, CA, USA); and incubated overnight with anti-RON, anti-D2-40 (Dako), and anti-CD34 (Abcam) antibodies in primary diluent solution (Invitrogen) at $4^{\circ} \mathrm{C}$. After washing in Tris-buffered saline Tween-20, the tissues were stained using the Dako Real ${ }^{\mathrm{TM}}$ EnVision HRP/DAB detection system and counterstained with hematoxylin. The stained tissues were scored and photographed using a light microscope.

Determination of RON expression. The immunostained specimens were examined by two dependent observers, unaware of the clinicopathological data. The staining intensities in cancer cells were graded as follows: 0 , no staining; 1 , weak; 2 , moderate; and 3, strong. The percentages of stained cancer cells were also graded as follows: 0 , none; $1,<10 \% ; 2,10-50 \%$; and $3,>50 \%$. The intensity score was multiplied by that of the percent staining to obtain an overall score. The mean overall score for the 88 tumors analyzed was 6.0 , which was selected as the cut-off for discriminating between RON expression levels. Specimens with scores $>6$ and $\leq 6$ were considered RON-positive and -negative, respectively.

Assessment of apoptosis in tissue samples. In order to detect and quantify apoptosis, the DeadEnd ${ }^{\mathrm{TM}}$ Colorimetric terminal deoxynucleotidyl transferase UTP nick-end labeling (TUNEL) system (Promega, Madison, WI, USA) was used according to the manufacturer's instructions. The number of positively stained cells with apoptotic morphology was enumerated in five random fields/sample. The apoptotic index (AI) was expressed as the number of positively stained nuclei, including apoptotic bodies among 1,000 tumor cell nuclei.

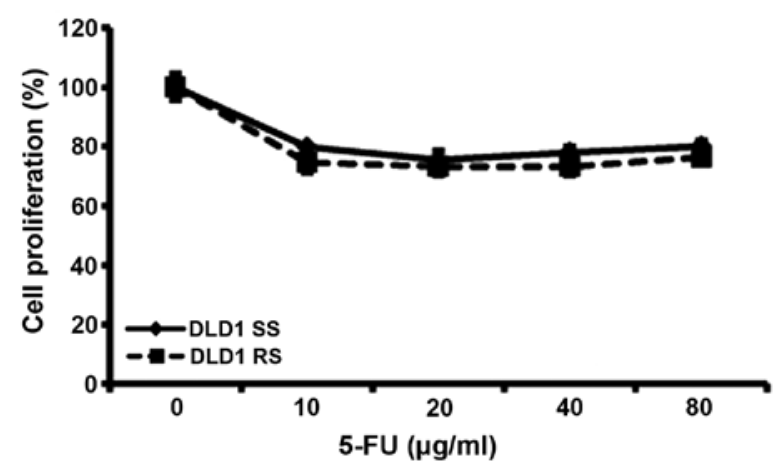

Figure 1. Cell viability assay of RON knocked-down colorectal cancer cells treated with 5-fluorouracil (FU) $(0,10,20,40$ or $80 \mu \mathrm{g} / \mathrm{ml})$. RON knockdown did not enhance 5-FU-induced death in DLD1 cells. RON, Recepteur d'Origine Nantais; SS, scramble siRNA; RS, RON-specific siRNA.

Determination of microvessel (MVD) and lymphatic vessel (LVD) densities. Microvessels and lymphatic vessels were detected using anti-CD34 and anti-D2-40 antibodies. MVD and LVD were quantified based on an international consensus. The stained sections were visualized at low magnification (x40) to identify the areas with high vascular density (hot spots) within the peritumoral and intratumoral regions. These high neovascular areas could occur anywhere within the tumor, but were most frequent at the margins of the carcinoma. Vessels in five areas with hot spots were enumerated at high magnification (x200). MVD and LVD were expressed as the mean number of vessels in these areas.

Statistical analyses. For intergroup comparisons, the Student's t-test was used to determine statistical significance, where data are presented as the mean $\pm \mathrm{SD}$. Correlations between various clinicopathological parameters and RON expression were performed using the $\chi^{2}$ and Fisher exact tests. The relationship of RON expression with AI, MVD or LVD was also evaluated by the Student's t-test. Actuarial survival rates of patients with either positive or negative RON expression were evaluated according to the Kaplan-Meier method, and the differences were tested with a log-rank test. The Cox regression model was used to determine the prognostic significance of each parameter by a multivariate analysis. The statistical analysis was performed with Statistical Package for the Social Sciences (SPSS/PC+ 15.0; SPSS, Inc., Chicago, IL, USA). P-values $<0.05$ were considered statistically significant.

\section{Results}

Effect of RON on chemosensitivity of colorectal cancer cell lines. In order to investigate the function of RON in the oncogenic behavior of colorectal cancer cells, we used RON-specific siRNAs to silence its endogenous gene expression in DLD1 cells. 5-FU is known to induce apoptosis and affect the cell cycle of these colorectal cancer cells (20-22). Cell viability assays revealed that RON knockdown did not enhance 5-FU-induced death in DLD1 cells (Fig. 1). We next performed flow cytometric analyses to evaluate the impact of RON on apoptosis and cell cycle distribution. The apoptotic rate of DLD1 cells upon RON knockdown significantly increased, 
A

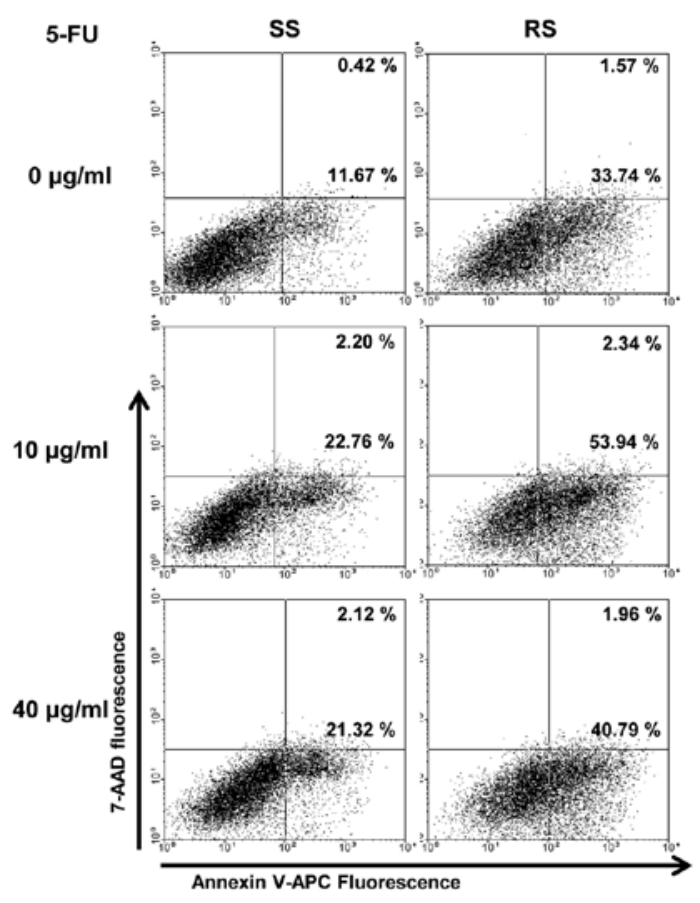

B

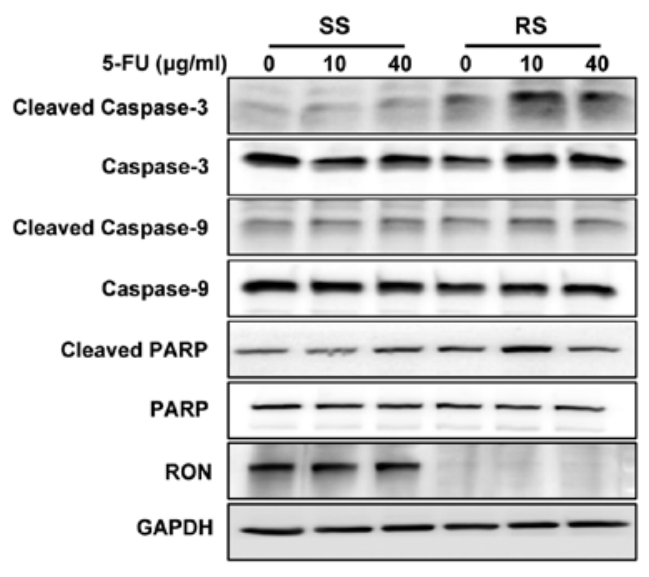

C

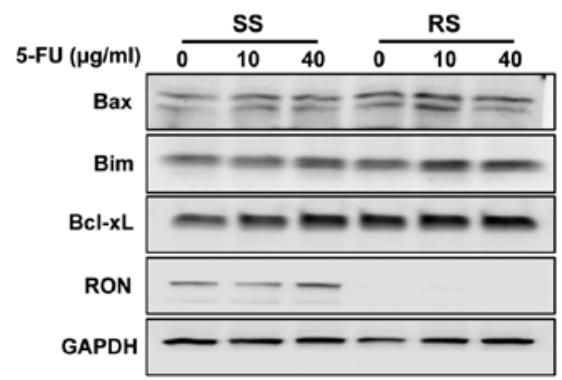

Figure 2. Effect of RON knockdown on apoptosis in colorectal cancer cells treated with 5-fluorouracil (FU) ( 0 , 10 or $40 \mu \mathrm{g} / \mathrm{ml})$. (A) RON knockdown promotes 5-FU-induced apoptosis in colorectal cancer cells. The proportion of apoptotic cells induced by transfection of RS was greater than that induced by transfection of SS in DLD1 cells with 5-FU treatment. (B) RON knockdown with 5-FU treatment induces apoptosis-related proteins of colorectal cancer cells. Cleaved caspase-3 and poly(ADP-ribose) polymerase (PARP) levels were increased in RS-transfected DLD1 cells treated with 5-FU, but that of cleaved caspase-9 remained unaffected. (C) RON knockdown along with 5-FU treatment induces apoptosis-regulatory proteins in colorectal cancer cells. Expression of Bax and Bim increased in RS-transfected DLD1 cells treated with 5-FU. Bcl-xL expression remained unaffected in DLD1 cells. RON, Recepteur d'Origine Nantais; SS, scramble siRNA; RS, RON-specific siRNA.

when compared with that in presence of scramble siRNAs (11.67 vs. $33.74 \%)$. It also augmented the 5-FU-induced apoptosis compared with that upon scramble siRNA treatment in DLD1 cells (22.76 and 21.32 vs. 53.94 and $40.79 \%$, respectively) (Fig. 2A). We further investigated caspase activities upon RON knockdown in these cell lines. Cleaved caspase-3 and PARP levels were upregulated in DLD1 cells following RON knockdown and 5-FU treatment (Fig. 2B). Fig. 2C illustrates that Bax and Bim protein levels increased in RON knocked down DLD1 cells. However, Bcl-xL level remained unaltered upon RON knockdown along with 5-FU treatment. However, this treatment arrested DLD1 cells in the subG1 and G0/G1 phases (Fig. 3A). Next, we evaluated the effect of RON knockdown on various cell cycle regulatory proteins. As Fig. 3B shows, cyclin B1 and D3, and CDK2 and 6 were significantly downregulated at the protein level. Moreover, the CDK inhibitor p21 was upregulated in the presence of RON-specific siRNAs and 5-FU in DLD1 cells. To evaluate whether RON affects the oncogenic behavior of colorectal cancer cells, migration and invasion assays were performed. The number of invading cells decreased in DLD1 cells treated with RON-specific siRNAs, with $10 \mu \mathrm{g} / \mathrm{ml} 5-\mathrm{FU}$ compared with scramble siRNA-treated controls $(\mathrm{P}=0.263$, 0.033 and 0.068 , respectively; Fig. 4A). The artificial wound gap was also significantly broader on plates containing RON knocked down DLD1 cells, with $10 \mu \mathrm{g} / \mathrm{ml} 5$-FU than that on control plates $(\mathrm{P}=0.218,0.020$ and 0.137 , respectively; Fig. 4B).

Effect of RON on the oncogenic signaling pathway in colorectal cancer cells. To examine whether RON activates intracellular signaling pathways in colorectal cancer cells, we measured the levels of phosphorylated $\beta$-catenin signaling proteins by western blotting. The phosphorylation level of $\beta$-catenin was increased by RON knockdown along with 5-FU treatment in DLD1 cells (Fig. 5).

Effect of RON on angiogenesis in colorectal cancer cells. We determined whether conditioned media (CM) from RON and scramble siRNA-transfected colorectal cancer cells affect angiogenesis in HUVECs by performing Matrigel invasion and endothelial tube formation assays. CM from RON knocked down DLD1 cells significantly inhibited endothelial tube formation compared with that from the respective mocktreated control cells ( $\mathrm{P}=0.045$; Fig. 6A). However, $\mathrm{CM}$ from RON knocked down DLD1 cells did not inhibit invasion of HUVECs as compared to the controls ( $\mathrm{P}=0.091$; Fig. 6B). The RON knockdown also decreased the expression of angiogenic inducers VEGF-A and HIF-1 $\alpha$, and increased that of the angiogenic inhibitor angiostatin in DLD1 cells (Fig. 6C). 
A

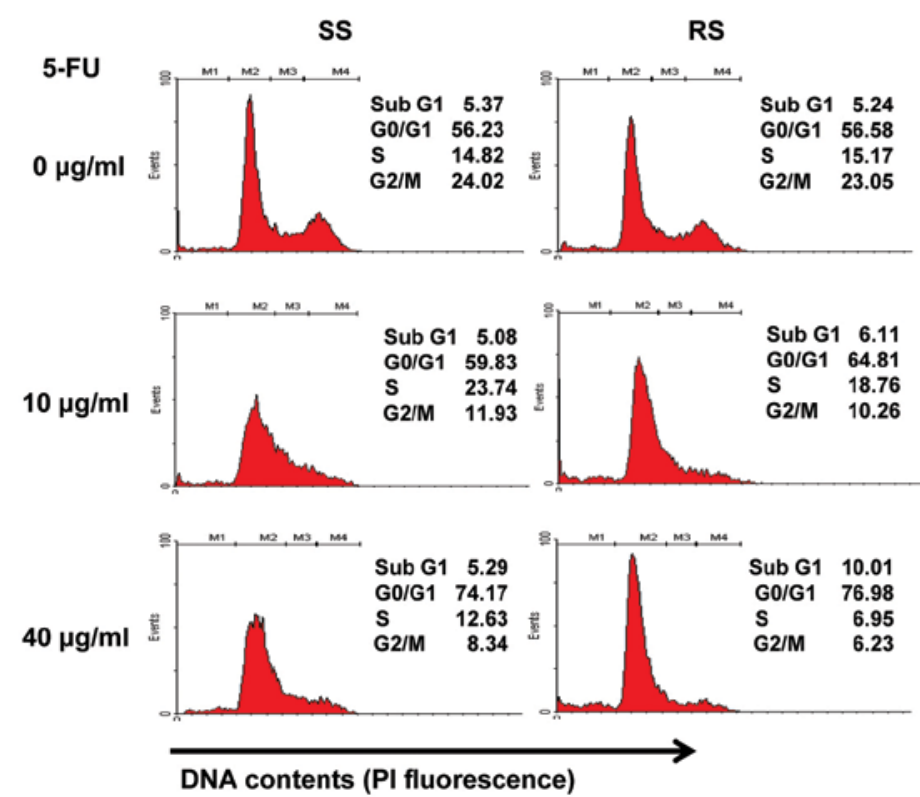

B

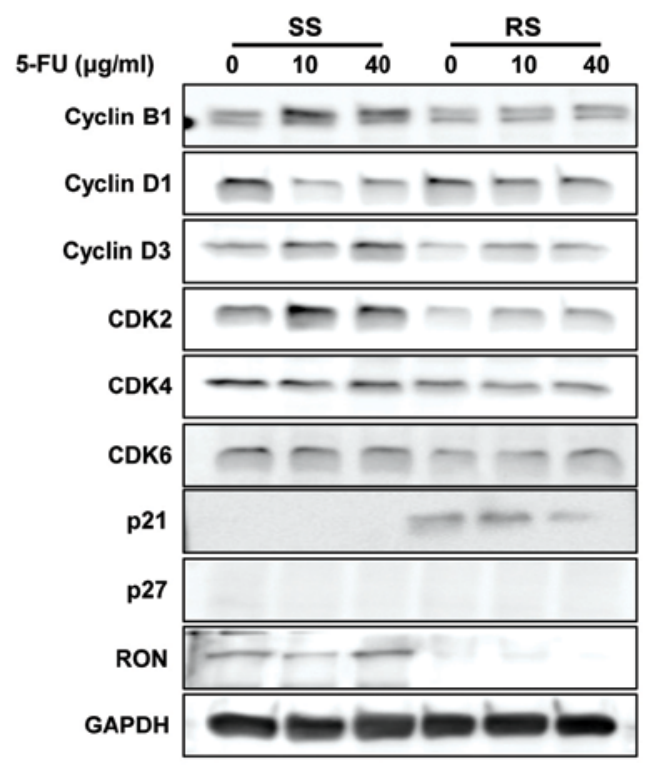

Figure 3. Effect of RON knockdown on cell cycle of colorectal cancer cells treated with 5-fluorouracil (FU) $(0,10 \mathrm{or} 40 \mu \mathrm{g} / \mathrm{ml})$. (A) RON knockdown promotes cell cycle arrest at the subG1 and G0/G1 phases in DLD1 cells treated with 5-FU. (B) RON knockdown along with 5-FU treatment regulates cell cycle-related proteins. Expression of cyclin B1 and D3, and CDK2 and 6 decreased, while that of p21 increased in RS-transfected DLD1 cells treated with 5-FU. RON, Recepteur d'Origine Nantais; SS, scramble siRNA; RS, RON-specific siRNA.
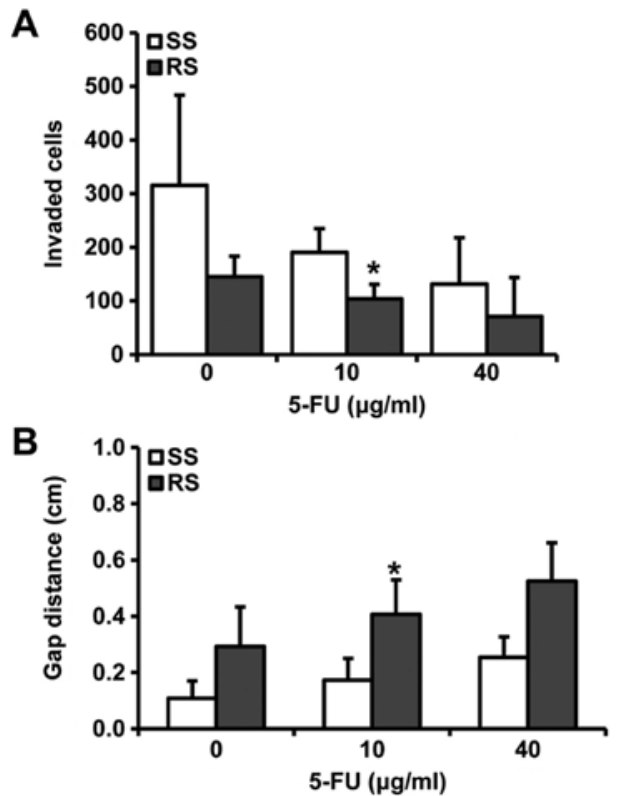

Figure 4. Effect of RON knockdown on invasion and migration of colorectal cancer cells treated with 5-fluorouracil (FU) (0, 10 or $40 \mu \mathrm{g} / \mathrm{ml})$. (A) The number of invading cells decreased in DLD1 cells treated with RON-specific siRNAs, with $10 \mu \mathrm{g} / \mathrm{ml} \mathrm{5-FU} \mathrm{compared} \mathrm{with} \mathrm{scramble} \mathrm{siRNA-treated}$ controls ( $\mathrm{P}=0.263,0.033$ and 0.068 , respectively). (B) The artificial wound gap was also significantly broader on plates containing RON knocked down DLD1 cells, with $10 \mu \mathrm{g} / \mathrm{ml} 5$-FU than that on control plates $(\mathrm{P}=0.218,0.020$ and 0.137 , respectively). Graphs for cell migration are represented as relative healing distances $\left({ }^{*} \mathrm{P}<0.05\right)$. RON, Recepteur d'Origine Nantais; SS, scramble siRNA; RS, RON-specific siRNA.

Collectively, these results suggest that RON is associated with angiogenesis and neovascularization in colorectal cancer cells.

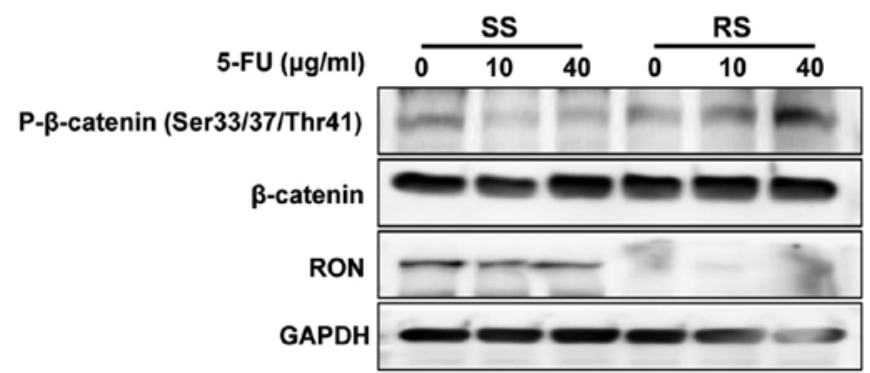

Figure 5. Effect of RON knockdown and 5-fluorouracil (FU) treatment (0, 10 or $40 \mu \mathrm{g} / \mathrm{ml}$ ) on oncogenic signaling pathway in colorectal cancer cells. Phosphorylation levels of $\beta$-catenin (Ser33/37/Thr41) was increased in RS-transfected DLD1 cells treated with 5-FU. RON, Recepteur d'Origine Nantais; SS, scrambled siRNA; RS, RON-specific siRNA.

Effect of RON on lymphangiogenesis in colorectal cancer cells. To examine the effects of RON on lymphangiogenesis in HLECs, we performed Matrigel invasion and tube formation assays using $\mathrm{CM}$ from RON and scramble siRNA-treated DLD1 cells. CM from RON knocked down DLD1 cells significantly inhibited lymphatic endothelial tube formation compared with that from the control cells $(\mathrm{P}=0.031$; Fig. 7A). However, CM from RON knocked down DLD1 cells failed to inhibit invasion of HLECs when compared with that from the respective controls ( $\mathrm{P}=0.325$; Fig. 7B). RON knockdown also suppressed expression of the lymphangiogenic inducers VEGF-C and COX-2 in DLD1 cells (Fig. 7C).

Correlation between RON and clinicopathological features of colorectal cancer. To study the role of RON in colorectal cancer progression, we measured the protein levels of RON in formalin-fixed, paraffin-embedded tissue blocks. These 
A

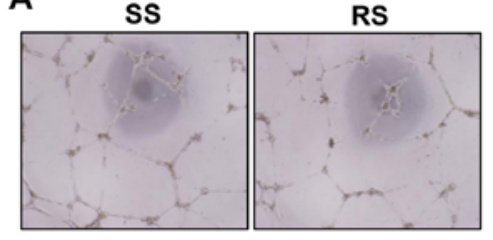

B
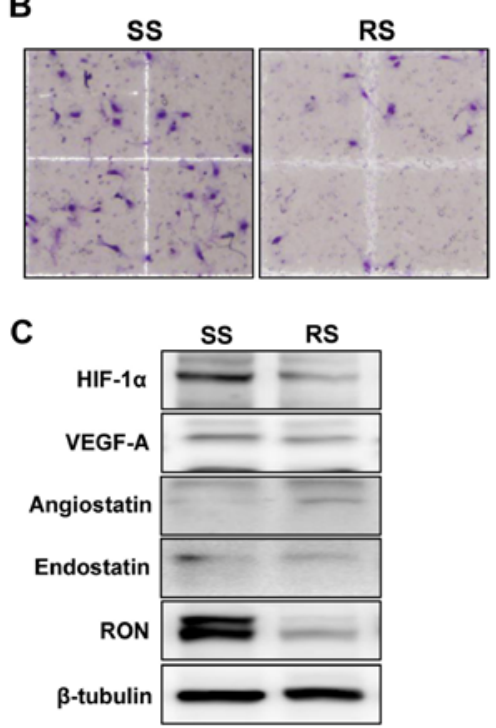

Figure 6. Effect of RON knockdown on angiogenesis in colorectal cancer cells. (A) The conditioned media (CM) from RS-transfected DLD1 cells significantly inhibited the formation of endothelial tubes compared with CM from SS-transfected cells ( $\mathrm{P}=0.045)$. (B) Matrigel invasion of HUVECs cultured in CM from RS-transfected DLD1 cells decreased compared with that of HUVECs cultured in CM from SS-transfected cells, though not significantly ( $\mathrm{P}=0.091)$. (C) RON knockdown decreased the expression of angiogenic inducers HIF- $1 \alpha$ and VEGF-A, and increased that of the angiogenic inhibitor angiostatin. RON, Recepteur d'Origine Nantais; SS, scramble siRNA; RS, RON-specific siRNA; HIF-1 $\alpha$, hypoxia-inducible factor- $1 \alpha$ VEGF, vascular endothelial growth factor; HUVECs, human umbilical vein endothelial cells. ${ }^{*} \mathrm{P}<0.05$ vs. SS.

tissues were obtained from 88 colorectal cancer patients whose clinicopathological data were available. We subsequently analyzed their survival rates, as well as the correlation between RON expression (determined by immunohistochemistry) and clinicopathological parameters. RON immunostaining was either absent or weak in normal colorectal mucosa, but predominant in the cytoplasm of cancer cells (Fig. 8). Moreover, it was undetected in the tumor stroma. The percentage of positively stained tumor cells and staining intensity for each sample were recorded. Of the 88 samples, RON-positivity was observed in 45 (51.1\%) tissues (Table I). Additionally, immunostaining of RON was significantly associated with age, tumor size, lymphovascular and perineural invasion, tumor stage and lymph node and distant metastasis $(\mathrm{P}=0.049,0.003$, $0.003,0.001,<0.001,<0.001$ and 0.001 , respectively; Table I). Moreover, the overall survival rate for patients positive for RON immunostaining was significantly lower than that for patients with negative immunostaining $(\mathrm{P}<0.001 ;$ Fig. 9).

Correlation of RON expression with apoptosis, angiogenesis, and lymphangiogenesis in colorectal cancers. All tumor samples were assessed by TUNEL assays and immunostaining
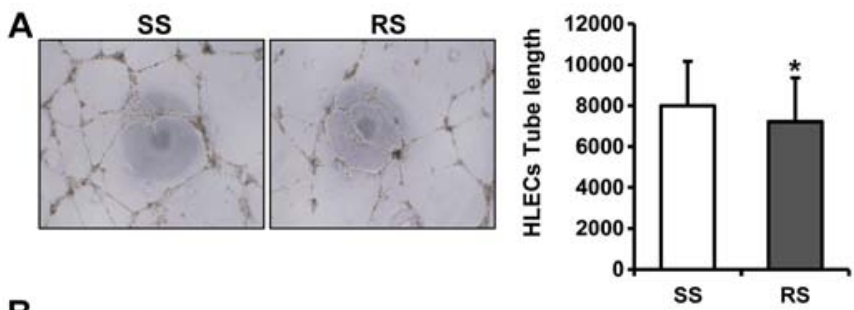

B
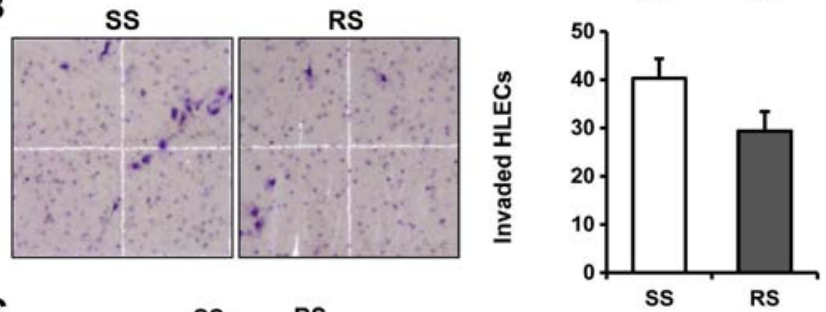

C

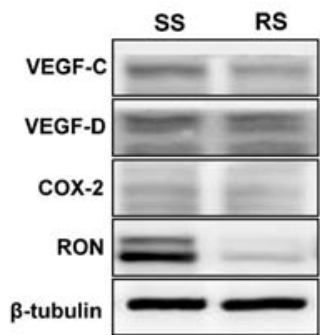

Figure 7. Effect of RON knockdown on lymphangiogenesis in colorectal cancer cells. (A) Tube formation in conditioned medium (CM) from RS-transfected DLD1 cells was significantly inhibited compared with that in CM from SS-transfected cells ( $\mathrm{P}=0.031)$. (B) Matrigel invasion of HLECs in CM from RS-transfected DLD1 cells decreased compared with that in CM from SS-transfected cells, but not significantly $(\mathrm{P}=0.325)$. (C) RON knockdown decreased the expression of lymphangiogenic inducers VEGF-C and COX-2 in DLD1 cells. RON, Recepteur d'Origine Nantais; SS, scramble siRNA; RS, RON-specific siRNA; VEGF, vascular endothelial growth factor; COX-2, cyclooxygenase-2; HLECs, human lymphatic endothelial cells. ${ }^{*} \mathrm{P}<0.05$ vs. SS.

for CD34 and D2-40 in order to determine apoptosis, angiogenesis and lymphangiogenesis. AI of the 88 tumor samples ranged from 0.6 to 30 , with a mean of 8.6 6.0. There was no significant difference observed between RON expression and AI ( $\mathrm{P}=0.752)$. Moreover, MVD values for the samples ranged from 23.0 to 429.0, with a mean of 116.0 76.4. The mean MVD value for RON-positive tumors was 133.982 .4 , significantly higher than that for RON-negative ones $(\mathrm{P}=0.040$; Table II). Furthermore, LVD values for the tumor samples ranged from 4.0 to 31.3 with a mean of 13.5 5.7. There was no significant difference between $R O N$ expression and LVD values $(\mathrm{P}=0.170$; Table II).

Correlation of AI, MVD, and LVD with clinicopathological features of colorectal cancer. The correlation of AI, MVD and LVD with clinicopathological parameters is summarized in Table III. Although LVD was associated with lymph node metastasis $(\mathrm{P}=0.041)$, no significant correlation was observed between either AI or MVD and various clinicopathological parameters (Table III).

\section{Discussion}

Overexpression and/or activation of RON has been implicated in the progression and metastasis of diverse epithelial cancers, 


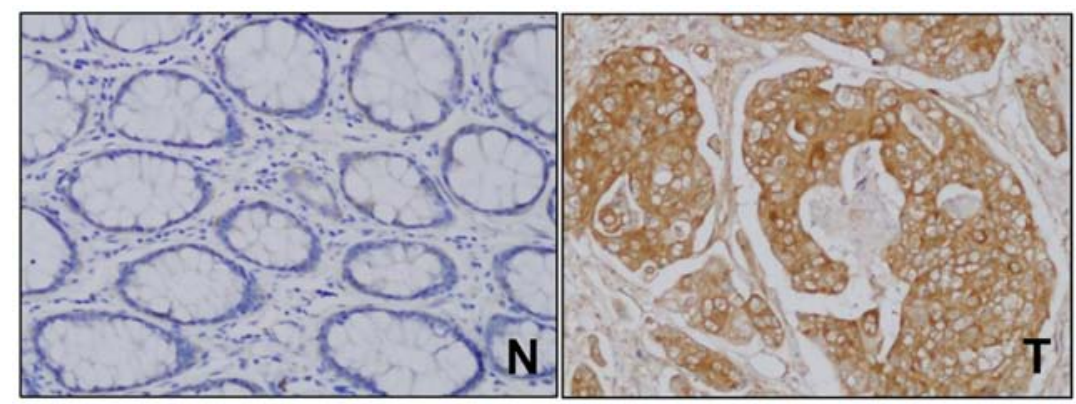

Figure 8. Expression of RON in colorectal cancer tissues. RON immunostaining was predominant in the cytoplasm of tumor cells, being either absent or weak in normal colon mucosa. Magnification, x200; RON, Recepteur d'Origine Nantais; N, paired normal colon mucosa tissue; T, colorectal cancer tissue.

Table I. Correlation between RON expression and the clinicopathological parameters of patients with colorectal cancer.

\begin{tabular}{|c|c|c|c|c|}
\hline & \multirow[b]{2}{*}{$\begin{array}{l}\text { Total } \\
(\mathrm{n}=88)\end{array}$} & \multicolumn{2}{|c|}{ RON } & \multirow[b]{2}{*}{ P-value } \\
\hline & & $\begin{array}{l}\text { Negative } \\
(n=43)\end{array}$ & $\begin{array}{l}\text { Positive } \\
(n=45)\end{array}$ & \\
\hline Age (years) & & & & 0.049 \\
\hline$<65.8$ & 38 & 14 & 24 & \\
\hline$\geq 65.8$ & 50 & 29 & 21 & \\
\hline Gender & & & & 0.859 \\
\hline Male & 52 & 25 & 27 & \\
\hline Female & 36 & 18 & 18 & \\
\hline Tumor size $(\mathrm{cm})$ & & & & 0.003 \\
\hline$<4.8$ & 45 & 29 & 16 & \\
\hline$\geq 4.8$ & 43 & 14 & 29 & \\
\hline Histologic type & & & & 0.687 \\
\hline Differentiated & 77 & 37 & 40 & \\
\hline Undifferentiated & 11 & 6 & 5 & \\
\hline $\begin{array}{l}\text { Lymphovascular } \\
\text { invasion }\end{array}$ & & & & 0.003 \\
\hline Negative & 58 & 35 & 23 & \\
\hline Positive & 30 & 8 & 22 & \\
\hline Perineural invasion & & & & 0.001 \\
\hline Negative & 63 & 38 & 25 & \\
\hline Positive & 25 & 5 & 20 & \\
\hline Stage & & & & $<0.001$ \\
\hline $\mathrm{I} / \mathrm{II}$ & 42 & 32 & 10 & \\
\hline III/IV & 46 & 11 & 35 & \\
\hline Depth of invasion (T) & & & & 0.068 \\
\hline $\mathrm{T} 1 / \mathrm{T} 2$ & 23 & 15 & 8 & \\
\hline $\mathrm{T} 3 / \mathrm{T} 4$ & 65 & 28 & 37 & \\
\hline $\begin{array}{l}\text { Lymph node } \\
\text { metastasis (N) }\end{array}$ & & & & $<0.001$ \\
\hline N0 & 45 & 33 & 12 & \\
\hline N1-3 & 43 & 10 & 33 & \\
\hline Distant metastasis (M) & & & & 0.001 \\
\hline M0 & 78 & 43 & 35 & \\
\hline M1 & 10 & 0 & 10 & \\
\hline
\end{tabular}

RON, Recepteur d'Origine Nantais.

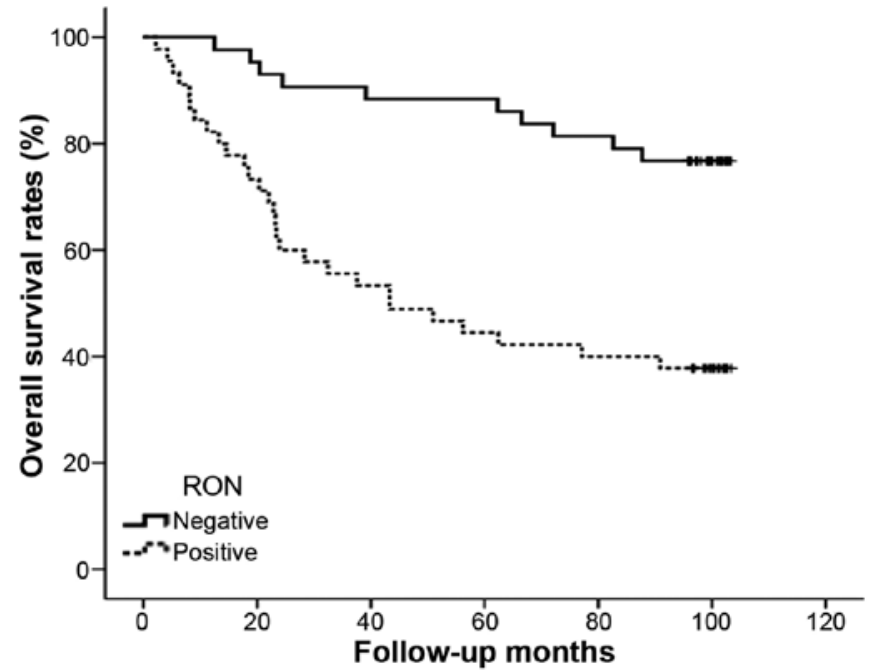

Figure 9. Kaplan-Meier survival curve correlating overall survival rate with negative and positive (solid and dotted lines, respectively) RON expression. The overall survival rate of patients positive for RON immunostaining was significantly lower than that of patients lacking RON-positive tumors $(\mathrm{P}<0.001)$. RON, Recepteur d'Origine Nantais.

Table II. Correlation of RON expression with tumor cell apoptosis, angiogenesis and lymphangiogenesis in colorectal cancer.

\begin{tabular}{lcccc}
\hline & & \multicolumn{2}{c}{ RON expression } & \\
\cline { 3 - 4 } Indices & Total & Negative & Positive & P-value \\
\hline AI & $8.6 \pm 6.0$ & $9.1 \pm 7.2$ & $8.2 \pm 4.7$ & 0.752 \\
MVD & $116.0 \pm 76.4$ & $97.3 \pm 65.5$ & $133.9 \pm 82.4$ & 0.040 \\
LVD & $13.5 \pm 5.7$ & $12.2 \pm 4.8$ & $15.3 \pm 6.4$ & 0.170 \\
\hline
\end{tabular}

RON, Recepteur d'Origine Nantais; AI, apoptotic index; MVD, microvessel density; LVD, lymphatic vessel density.

where it plays a causal role in tumor progression by promoting growth, survival and motility of tumor cells (23-29).

Cancer cells are typically characterized by increased resistance to apoptosis and cell cycle control $(5,6)$. In this context, 5-FU is known to induce apoptosis and affects the cell cycle of cancer cells (19). It is a widely used first-line drug 
Table III. Correlation of AI, MVD and LVD with clinicopathological parameters of patients with colorectal cancer.

\begin{tabular}{|c|c|c|c|c|c|c|}
\hline Parameters & $\mathrm{AI}($ mean $\pm \mathrm{SD})$ & P-value & $\operatorname{MVD}($ mean $\pm \mathrm{SD})$ & P-value & $\mathrm{LVD}($ mean $\pm \mathrm{SD})$ & P-value \\
\hline Stage & & 0.179 & & 0.098 & & 0.062 \\
\hline $\mathrm{I} / \mathrm{II}$ & $10.4 \pm 7.6$ & & $105.6 \pm 70.0$ & & $12.2 \pm 4.4$ & \\
\hline III/IV & $7.3 \pm 4.1$ & & $125.6 \pm 81.5$ & & $14.7 \pm 6.5$ & \\
\hline Depth of invasion (T) & & 0.549 & & 0.297 & & 0.222 \\
\hline $\mathrm{T} 1 / \mathrm{T} 2$ & $11.5 \pm 10.1$ & & $130.8 \pm 84.8$ & & $11.4 \pm 3.9$ & \\
\hline $\mathrm{T} 3 / \mathrm{T} 4$ & $7.8 \pm 4.2$ & & $110.8 \pm 73.3$ & & $14.2 \pm 6.1$ & \\
\hline LN metastasis $(\mathrm{N})$ & & 0.274 & & 0.232 & & 0.041 \\
\hline No & $10.4 \pm 7.6$ & & $108.3 \pm 71.7$ & & $12.2 \pm 4.4$ & \\
\hline $\mathrm{N} 1-3$ & $7.3 \pm 4.1$ & & $124.1 \pm 81.2$ & & $14.7 \pm 6.5$ & \\
\hline Distant metastasis (M) & & 0.389 & & 0.795 & & 0.102 \\
\hline M0 & $8.7 \pm 6.4$ & & $113.2 \pm 71.6$ & & $13.4 \pm 5.9$ & \\
\hline M1 & $8.4 \pm 2.9$ & & $138.4 \pm 109.4$ & & $15.1 \pm 3.5$ & \\
\hline
\end{tabular}

SD, standard deviation; AI, apoptotic index; MVD, microvessel density; LVD, lymphatic vessel density.

for colorectal cancer treatment, but limited by drug resistance and severe toxicity $(38,39)$.

In the present study, we evaluated whether RON knockdown affects chemosensitivity of colorectal cancer cells. Apoptosis is a highly regulated process, and various proapoptotic and anti-apoptotic genes are involved in this process $(40,41)$. Our study showed that RON knockdown enhances 5-FU-induced apoptosis by upregulating the activities of caspase-3 and PARP; as well as the expression of proapoptotic genes encoding Bax and Bim. In contrast, cell cycle progression is regulated by positive (cyclins and CDKs) and negative (CDKIs) effectors (42-44). Moreover, we showed that RON knockdown augments 5-FU-induced cell cycle arrest by decreasing the expression of cyclin B1 and D3, CDK2 and CDK6 and by inducing that of $\mathrm{p} 21$. Furthermore, regulation of cell migration, invasion and proliferation is crucial for maintaining cellular homeostasis, and its loss is a hallmark of cancer cells $(5,6)$. In our study, RON knockdown enhanced the 5-FU-induced inhibitory effect on the invasion and migration of colorectal cancer cells. These results suggest that RON functions to resist 5-FU sensitivity by inhibiting apoptosis, cell cycle arrest, invasion and migration in colorectal cancer cells.

To elucidate the mechanisms underlying the effects of RON knockdown observed in the present study, we investigated the intracellular signaling pathway that leads to inhibition of apoptosis, cell cycle arrest, migration and invasion. We found that the $\beta$-catenin signaling cascade is blocked upon RON knockdown along with 5-FU treatment. Previous studies have presented this cascade as one of the oncogenic signaling pathways activated by RON $(29,45,46)$. Therefore, our results suggest that RON may regulate 5-FU sensitivity of colorectal cancer cells via the $\beta$-catenin signaling cascade.

Angiogenesis and lymphangiogenesis are critical features of tumor growth and metastasis (7-11). Increasing evidence from in vitro and in vivo studies has shown that angiogenesis and lymphangiogenesis are associated with tumor growth, progression and poor clinical outcome in colorectal cancer (12-14). In the present study, we examined the impact of RON expression on angiogenic and lymphangiogenic phenotypes using in vitro assays. RON knockdown decreased umbilical vein endothelial tube formation and VEGF-A and HIF-1 $\alpha$ expression; and increased angiostatin expression in colorectal cancer cells. Previous studies showed that RON modulates angiogenic chemokine production and subsequent endothelial recruitment in prostatic cancers (47). Together, these results indicate that RON plays an important role in tumor progression by stimulating angiogenesis, and angiogenic and angiostatic factors in colorectal cancer.

In addition, RON knockdown decreased lymphatic endothelial cell tube formation and the expression of VEGF-C and COX-2 in colorectal cancer cells. Previous studies reported that VEGF-C, -D and COX-2 are associated with tumor lymphangiogenesis and lymph node metastasis $(9-11,48)$. Therefore, we suggest that RON contributes to tumor lymphangiogenesis in colorectal cancer.

RON is overexpressed in several cancer types and is associated with tumor progression (30-37). We next examined RON expression in a well-defined series of colorectal cancer tissue samples, with special reference to patient prognosis. RON expression was significantly increased in the cancer tissues compared with that in normal colorectal mucosa. It was also associated with age, tumor size and stage, lymphovascular and perineural invasion, lymph node and distant metastasis, and poor survival. Previous studies have associated RON expression with aggressive disease and poor clinical outcome in colorectal cancer patients $(30,31)$. RON expression has also been associated with progression and poor prognosis of gastric, ovarian and bladder cancers $(32,33,49,50)$. Our results, along with these studies, suggest that RON expression may help predict poor clinical outcome of colorectal cancer.

Finally, we evaluated the correlation of RON expression with tumor cell apoptosis, angiogenesis and lymphangiogenesis in colorectal cancer tissues, in order to confirm our in vitro observations. The mean MVD value for RON-positive tumors was significantly higher than that of RON-negative 
ones. However, there was no significant difference of RON expression with either AI or LVD.

In conclusion, RON influences tumor progression by inhibiting chemosensitivity and enhancing angiogenesis in colorectal cancer.

\section{References}

1. Brenner H, Kloor M and Pox CP: Colorectal cancer. Lancet 383 : 1490-1502, 2014

2. Park SH, Song CW, Kim YB, Kim YS, Chun HR, Lee JH, Seol WJ, Yoon HS, Lee MK, Lee JH, et al: Clinicopathological characteristics of colon cancer diagnosed at primary health care institutions. Intest Res 12: 131-138, 2014.

3. Lee CK: Clinicopathological characteristics of newly diagnosed colorectal cancers in community gastroenterology practice. Intest Res 12: 87-89, 2014.

4. Kim ER and Kim YH: Clinical application of genetics in management of colorectal cancer. Intest Res 12: 184-193, 2014.

5. Riethdorf S, Wikman H and Pantel K: Review: Biological relevance of disseminated tumor cells in cancer patients. Int J Cancer 123: 1991-2006, 2008.

6. Chambers AF, Groom AC and MacDonald IC: Dissemination and growth of cancer cells in metastatic sites. Nat Rev Cancer 2 563-572, 2002

7. Mittal K, Ebos J and Rini B: Angiogenesis and the tumor microenvironment: Vascular endothelial growth factor and beyond. Semin Oncol 41: 235-251, 2014.

8. Zhao Y and Adjei AA: Targeting angiogenesis in cancer therapy: Moving beyond vascular endothelial growth factor Oncologist 20: 660-673, 2015.

9. Gomes FG, Nedel F, Alves AM, Nör JE and Tarquinio SB: Tumor angiogenesis and lymphangiogenesis: Tumor/endothelia crosstalk and cellular/microenvironmental signaling mechanisms. Life Sci 92: 101-107, 2013.

10. Stacker SA, Williams SP, Karnezis T, Shayan R, Fox SB and Achen MG: Lymphangiogenesis and lymphatic vessel remodelling in cancer. Nat Rev Cancer 14: 159-172, 2014.

11. Duong T, Koopman P and Francois M: Tumor lymphangiogenesis as a potential therapeutic target. J Oncol 2012: 204946, 2012.

12. Marques I, Araújo A and de Mello RA: Anti-angiogenic therapies for metastatic colorectal cancer: Current and future perspectives. World J Gastroenterol 19: 7955-7971, 2013.

13. Royston D and Jackson DG: Mechanisms of lymphatic metastasis in human colorectal adenocarcinoma. J Pathol 217: 608-619, 2009.

14. Sun XF and Zhang H: Clinicopathological significance of stromal variables: Angiogenesis, lymphangiogenesis, inflammatory infiltration, MMP and PINCH in colorectal carcinomas. Mol Cancer 5: 43, 2006.

15. Zhang W, Meng Y, Liu N, Wen XF and Yang T: Insights into chemoresistance of prostate cancer. Int J Biol Sci 11: 1160-1170, 2015.

16. Ojha R, Bhattacharyya S and Singh SK: Autophagy in cancer stem cells: A potential link between chemoresistance, recurrence, and metastasis. Biores Open Access 4: 97-108, 2015.

17. Wang X, Li X, Fu X, Bai M, Li X, Mei Q, Nie J, Wu Z and Han W: Eliminating ovarian cancer stem cells: A potential therapeutic target for ovarian cancer chemoresistance. Curr Protein Pept Sci 16: 270-278, 2015

18. Carta A, Chetcuti R and Ayers D: An introspective update on the influence of miRNAs in breast carcinoma and neuroblastoma chemoresistance. Genet Res Int 2014: 743050, 2014.

19. Hoshiba T and Tanaka M: Optimization of the tissue source, malignancy, and initial substrate of tumor cell-derived matrices to increase cancer cell chemoresistance against 5-fluorouracil. Biochem Biophys Res Commun 457: 353-357, 2015.

20. Garza-Treviño EN, Said-Fernández SL and MartínezRodríguez HG: Understanding the colon cancer stem cells and perspectives on treatment. Cancer Cell Int 15: 2, 2015

21. Paldino E, Tesori V, Casalbore P, Gasbarrini A and Puglisi MA: Tumor initiating cells and chemoresistance: Which is the best strategy to target colon cancer stem cells? Biomed Res Int 2014: 859871, 2014.

22. Grossi V, Peserico A, Tezil T and Simone C: p38 $\alpha$ MAPK pathway: A key factor in colorectal cancer therapy and chemoresistance. World J Gastroenterol 20: 9744-9758, 2014.
23. Lu Y, Yao HP and Wang MH: Multiple variants of the RON receptor tyrosine kinase: Biochemical properties, tumorigenic activities, and potential drug targets. Cancer Lett 257: 157-164, 2007.

24. Leonis MA, Thobe MN and Waltz SE: Ron-receptor tyrosine kinase in tumorigenesis and metastasis. Future Oncol 3: 441-448, 2007.

25. Camp ER, Liu W, Fan F, Yang A, Somcio R and Ellis LM: RON, a tyrosine kinase receptor involved in tumor progression and metastasis. Ann Surg Oncol 12: 273-281, 2005.

26. Wang MH, Yao HP and Zhou YQ: Oncogenesis of RON receptor tyrosine kinase: A molecular target for malignant epithelial cancers. Acta Pharmacol Sin 27: 641-650, 2006.

27. Wang MH, Wang D and Chen YQ: Oncogenic and invasive potentials of human macrophage-stimulating protein receptor, the RON receptor tyrosine kinase. Carcinogenesis 24: 1291-1300, 2003.

28. Wagh PK, Peace BE and Waltz SE: Met-related receptor tyrosine kinase Ron in tumor growth and metastasis. Adv Cancer Res 100: $1-33,2008$

29. Danilkovitch-Miagkova A: Oncogenic signaling pathways activated by RON receptor tyrosine kinase. Curr Cancer Drug Targets 3: 31-40, 2003.

30. Lee CT, Chow NH, Su PF, Lin SC, Lin PC and Lee JC: The prognostic significance of RON and MET receptor coexpression in patients with colorectal cancer. Dis Colon Rectum 51: 1268-1274, 2008.

31. Park YL, Lee GH, Kim KY, Myung E, Kim JS, Myung DS, Park KJ, Cho SB, Lee WS, Jung YD, et al: Expression of RON in colorectal cancer and its relationships with tumor cell behavior and prognosis. Tumori 98: 652-662, 2012.

32. Song YA, Park YL, Kim KY, Myung E, Chung CY, Cho SB, Lee WS, Jung YD, Kweon SS and Joo YE: RON is associated with tumor progression via the inhibition of apoptosis and cell cycle arrest in human gastric cancer. Pathol Int 62: 127-136, 2012.

33. Maggiora P, Marchio S, Stella MC, Giai M, Belfiore A, De Bortoli M, Di Renzo MF, Costantino A, Sismondi P and Comoglio PM: Overexpression of the RON gene in human breast carcinoma. Oncogene 16: 2927-2933, 1998.

34. Kim SA, Yoon TM, Lee DH, Park YL, Lee KH, Lim SC, Joo YE and Lee JK: RON (recepteur d'origine Nantais) expression and its association with tumor progression in laryngeal squamous cell carcinoma. Auris Nasus Larynx 41: 201-206, 2014.

35. Yoon TM, Kim SA, Park YL, Lee KH, Sung MW, Lee JK, Lim SC, Chung IJ and Joo YE: Expression of the receptor tyrosine kinase recepteur d'origine Nantais and its association with tumor progression in hypopharyngeal cancer. Head Neck 35: 1106-1113, 2013.

36. Chung CY, Park YL, Song YA, Myung E, Kim KY, Lee GH, Ki HS, Park KJ, Cho SB, Lee WS, et al: Knockdown of RON inhibits AP-1 activity and induces apoptosis and cell cycle arrest through the modulation of Akt/FoxO signaling in human colorectal cancer cells. Dig Dis Sci 57: 371-380, 2012.

37. Cho SB, Park YL, Song YA, Kim KY, Lee GH, Cho DH, Myung DS, Park KJ, Lee WS, Chung IJ, et al: Small interfering RNA-directed targeting of RON alters invasive and oncogenic phenotypes of human hepatocellular carcinoma cells. Oncol Rep 26: 1581-1586, 2011.

38. American Joint Committee on Cancer Classification (AJCC): Cancer Staging Manual. 6th edition, revised. Lippincott-Raven, Philadelphia, pp113-123, 2002.

39. Paschall AV and Liu K: Epigenetic regulation of apoptosis and cell cycle regulatory genes in human colon carcinoma cells. Genom Data 5: 189-191, 2015.

40. Mason EF and Rathmell JC: Cell metabolism: An essential link between cell growth and apoptosis. Biochim Biophys Acta 1813: 645-654, 2011.

41. Schultz DR and Harrington WJ Jr: Apoptosis: Programmed cell death at a molecular level. Semin Arthritis Rheum 32: 345-369, 2003.

42. Morgan DO: Principles of CDK regulation. Nature 374: 131-134, 1995.

43. Graña X and Reddy EP: Cell cycle control in mammalian cells: Role of cyclins, cyclin dependent kinases (CDKs), growth suppressor genes and cyclin-dependent kinase inhibitors (CKIs). Oncogene 11: 211-219, 1995.

44. Johnson N and Shapiro GI: Cyclin-dependent kinases (cdks) and the DNA damage response: Rationale for cdk inhibitorchemotherapy combinations as an anticancer strategy for solid tumors. Expert Opin Ther Targets 14: 1199-1212, 2010. 
45. Wang D, Shen $\mathrm{Q}, \mathrm{Xu} \mathrm{XM}$, Chen $\mathrm{YQ}$ and Wang $\mathrm{MH}$ : Activation of the RON receptor tyrosine kinase attenuates transforming growth factor-betal-induced apoptotic death and promotes phenotypic changes in mouse intestinal epithelial cells. Carcinogenesis 26: 27-36, 2005.

46. Danilkovitch-Miagkova A, Miagkov A, Skeel A, Nakaigawa N, Zbar B and Leonard EJ: Oncogenic mutants of RON and MET receptor tyrosine kinases cause activation of the beta-catenin pathway. Mol Cell Biol 21: 5857-5868, 2001.

47. Thobe MN, Gurusamy D, Pathrose P and Waltz SE: The Ron receptor tyrosine kinase positively regulates angiogenic chemokine production in prostate cancer cells. Oncogene 29: 214-226, 2010
48. Karnezis T, Shayan R, Fox S, Achen MG and Stacker SA: The connection between lymphangiogenic signalling and prostaglandin biology: A missing link in the metastatic pathway. Oncotarget 3: 893-906, 2012.

49. Maggiora P, Lorenzato A, Fracchioli S, Costa B, Castagnaro M, Arisio R, Katsaros D, Massobrio M, Comoglio PM and Flavia Di Renzo M: The RON and MET oncogenes are co-expressed in human ovarian carcinomas and cooperate in activating invasiveness. Exp Cell Res 288: 382-389, 2003.

50. Cheng HL, Liu HS, Lin YJ, Chen HH, Hsu PY, Chang TY, Ho CL, Tzai TS and Chow NH: Co-expression of RON and MET is a prognostic indicator for patients with transitional-cell carcinoma of the bladder. Br J Cancer 92: 1906-1914, 2005. 